\title{
Impacto da implementação do serviço de residência em cirurgia e traumatologia buco-maxilo-facial em um hospital público em Campina Grande-PB
}

\author{
Impact of the implementation of the residency service in surgery and \\ oral-maxillo-facial traumatology in a public hospital in Campina Grande-PB \\ Impacto de la implementación del servicio de residencia en cirugía y \\ traumatología oral-maxilofacial en un hospital público de Campina Grande-PB
}

Recebido: 29/06/2021 | Revisado: 03/07/2021 | Aceito: 05/07/2021 | Publicado: 06/07/2021

\author{
Reginaldo Fernandes da Silva \\ ORCID: https://orcid.org/0000-0002-1734-6150 \\ Hospital de Emergência e Trauma Dom Luiz Gonzaga Fernandes, Brasil \\ E-mail: regis.ferns@hotmail.com \\ Gustavo José de Luna Campos \\ ORCID: https://orcid.org/0000-0003-3540-8084 \\ Hospital de Emergência e Trauma Dom Luiz Gonzaga Fernandes, Brasil \\ E-mail: camposctbmf@yahoo.com.br \\ Alfredo Lucas Neto \\ ORCID: https://orcid.org/0000-0002-6849-0240 \\ Hospital de Emergência e Trauma Dom Luiz Gonzaga Fernandes, Brasil \\ E-mail:dr.alfredolucas@gmail.com \\ Hécio Henrique de Araújo Morais \\ ORCID: https://orcid.org/0000-0002-6450-1483 \\ Universidade Federal do Rio Grande do Norte, Brasil \\ E-mail: heciomorais@hotmail.com \\ Nilton Freitas Medrado Filho \\ ORCID: https://orcid.org/0000-0003-1090-4727 \\ Universidade do Estado do Rio Grande do Norte, Brasil \\ E-mail: niltonfreitasfilho@hotmail.com \\ André Vajgel Fernandes \\ ORCID: https://orcid.org/0000-0003-1534-7595 \\ Hospital de Emergência e Trauma Dom Luiz Gonzaga Fernandes, Brasil \\ E-mail: avajgel@hotmail.com
}

\begin{abstract}
Resumo
O objetivo foi avaliar o impacto da implementação do serviço de residência em cirurgia e traumatologia buco-maxilofacial em um hospital público em Campina Grande-PB, Brasil. Foram analisados 516 prontuários de pacientes operados no Hospital de Emergência e Trauma Dom Luiz Gonzaga Fernandes (HETDLGF), no período de março de 2017 a fevereiro de 2018 (período sem o serviço de residência) e março de 2018 a fevereiro de 2019 ( $1^{\circ}$ ano do serviço de residência). Os resultados foram compilados em um banco de dados organizado em planilhas do Microsoft Excel® e posteriormente analisados no software SPSS $®$ for Windows 25.0. Dentre os 516 pacientes, 236 foram operados no ano antes da residência (média de 19,6 cirurgias mensais) e 280 pacientes foram operados no ano da residência (média de 23,3 cirurgias por mês). Observa-se um acréscimo de 44 cirurgias, equivalendo a mais de dois meses de cirurgias a mais em relação ao ano antecessor à implementação da residência. $\mathrm{O}$ tempo médio de espera pelo paciente para a cirurgia diminuiu em 2,5 dias e o tempo médio de internação hospitalar em 2,6 dias. O hospital obteve uma economia média de $\mathrm{R}$ \$ 1.102,58 por internação, em comparação aos anos anteriores. Da amostra total, $416(80,6 \%)$ eram do sexo masculino e $100(19,4 \%)$ eram do sexo feminino. Conclui-se que a implementação de um serviço de residência em um hospital público promove um impacto positivo, reduzindo o tempo de espera do paciente pelo tratamento cirúrgico, o tempo de internação hospitalar e consequentemente, os custos financeiros ao hospital.
\end{abstract}

Palavras-chave: Lesões Faciais; Cirurgiões Bucomaxilofaciais; Epidemiologia.

\begin{abstract}
The objective was to evaluate the impact of the implementation of the residency service in oral and maxillofacial surgery and traumatology in a public hospital in Campina Grande - PB, Brazil. 516 medical records were analyzed of patients operated at the Hospital de Emergência e Trauma Dom Luiz Gonzaga Fernandes (HETDLGF), from March 2017 to February 2018 (period without the residency service) and March 2018 to February 2019 (1st year residence service). The results were compiled into a database organized in Microsoft Excel $\AA$ spreadsheets and later analyzed in the SPSS $₫$
\end{abstract}


software for Windows 25.0. Among the 516 patients, 236 were operated in the year before residence (mean 19.6 surgeries monthly) and 280 patients were operated in the year of residence (mean 23.3 surgeries per month). It is observed an increase of 44 surgeries, equivalent to more than two months of surgeries compared to the year preceding the implementation of the residency. The average waiting time for the patient for surgery decreased by 2.5 days and the average hospital stay by 2.6 days. The hospital achieved an average savings of $\mathrm{R} \$ 1,102.58$ per admission, compared to previous years. Of the total sample, $416(80.6 \%)$ were male and $100(19.4 \%)$ were female. It is concluded that the implementation of a residency service in a public hospital promotes a positive impact, reducing the patient's waiting time for surgical treatment, the length of hospital stay and, consequently, the financial costs to the hospital.

Keywords: Facial Injuries; Oral and Maxillofacial Surgeons; Epidemiology.

\section{Resumen}

El objetivo fue evaluar el impacto de la implementación del servicio de residencia en cirugía y traumatología oral y maxilofacial en un hospital público de Campina Grande-PB, Brasil. Se analizaron un total de 516 historias clínicas de pacientes intervenidos en el Hospital de Emergencias y Traumatismos Dom Luiz Gonzaga Fernandes (HETDLGF) desde marzo de 2017 a febrero de 2018 (período sin servicio de residencia) y de marzo de 2018 a febrero de 2019 (1er año de la servicio de residencia). Los resultados se recopilaron en una base de datos organizada en hojas de cálculo de Microsoft Excel® y luego se analizaron con el software SPSS ${ }^{\circledR}$ para Windows 25.0. Entre los 516 pacientes, 236 fueron operados en el año anterior a la residencia (media de 19,6 cirugías mensuales) y 280 pacientes fueron operados en el año de residencia (media de 23,3 cirugías por mes). Hay un aumento de 44 cirugías, equivalente a más de dos meses de cirugías en comparación con el año anterior a la implementación de la residencia. El tiempo medio de espera del paciente para la cirugía se redujo en 2,5 días y la estancia hospitalaria media en 2,6 días. El hospital logró un ahorro promedio de R \$ 1.102,58 por ingreso, en comparación con años anteriores. Del total de la muestra, 416 (80,6\%) eran hombres y $100(19,4 \%)$ eran mujeres. Se concluye que la implementación de un servicio de residencia en un hospital público promueve un impacto positivo, reduciendo el tiempo de espera del paciente para el tratamiento quirúrgico, la estancia hospitalaria y, en consecuencia, los costos económicos al hospital.

Palabras clave: Lesiones faciales; Cirujanos orales y maxilofaciales; Epidemiología.

\section{Introdução}

Os traumas faciais têm grande destaque nos atendimentos de emergências gerais, tendo a face uma área de grande exposição e expressão facial, e, por isso mais exposta ao trauma (Wulkan, Parreira, Botter, 2005). A desfiguração facial decorrente do trauma de face convida a uma resposta social geralmente negativa que leva a um impacto prejudicial sobre o estado psicológico de um indivíduo (Sahni, 2017), gerando possíveis sequelas faciais permanentes, podendo comprometer, definitivamente, a vida do ser humano, marginalizando o indivíduo de interações sociais (Falcão et al., 2005). Dessa forma, nas emergências gerais, deve-se preconizar um atendimento sistematizado e multidisciplinar para possibilitar a correta sequência de atendimento para essas vítimas de traumas faciais, desde os casos mais graves aos mais brandos (Motta, 2009).

O Hospital de Emergência e Trauma Dom Luiz Gonzaga Fernandes (HETDLGF), situado na cidade de Campina Grande no estado da Paraíba (Brasil), integra a rede hospitalar do Governo estadual. A unidade é referência em trauma para 203 municípios da Paraíba, além de alguns municípios do Rio Grande do Norte, Pernambuco e Ceará (Paraíba, 2019).

Nos primeiros cinco meses de 2019, foram atendidos 42.763 pacientes e realizadas 3.868 cirurgias. Em 2018, no mesmo período foram prestados 38.324 atendimentos e realizadas 3.708 cirurgias, em diversas especialidades (Paraíba, 2019).

Devido ao aumento das mortes e incapacidades ocorridas por fatores externos havendo comprometimento da face, o atendimento odontológico hospitalar está cada vez mais voltado para esta etiologia (Rodrigues et al., 2006). Acidentes que comprometem a face constituem, portanto, a primeira causa de mortalidade de jovens até 29 anos e a terceira maior causa de morte entre pessoas de 30 a 44 anos. Durante o ano de 2000, foram responsáveis por 29.640 mortes, inferior apernas às mortes causadas por doenças cardiovasculares (Santos et al., 2016).

É incontestável a importância de um serviço de Cirurgia e Traumatologia Buco-Maxilo-Facial (CTBMF) nos grandes hospitais especializados em trauma, uma vez que as fraturas faciais representam um segmento importante dos problemas de saúde pública devido ao fato de suas sequelas poderem resultar em sérias alterações estéticas e funcionais (Leite Segundo et al., 2005). O tratamento dessas lesões visa à recuperação da função, com o mínimo de alteração estética do paciente (Rodrigues et 
al., 2006).

Desta forma, a construção de um banco de dados possibilita uma comparação entre diversas regiões e até mesmo dentro de um mesmo serviço. Além disso, estudos comparativos sobre os traumas faciais permitem analisar a mudança do perfil epidemiológico dos pacientes, sendo possível programar medidas adequadas de prevenção e tratamento, além de se observar o reflexo de certas mudanças sociais, culturais e de legislação (Lee, 2012).

Para tanto, o presente estudo se propôs avaliar o impacto da implementação do serviço de Residência em Cirurgia e Traumatologia Buco-Maxilo-Facial em um Hospital Público na cidade de Campina Grande-PB.

\section{Metodologia}

Trata-se de um estudo quantitativo, descritivo, retrospectivo e documental (Gil, 2008), onde foi desenvolvido após o conhecimento do Comitê Científico do hospital e aprovação em Comitê de Ética em Pesquisa do Hospital Agamenon Magalhães (HAM), sob o parecer no 3.949.209 de acordo com a resolução n. ${ }^{\circ}$ 466/12 do Conselho Nacional de Saúde do Ministério da Saúde, solicitada a dispensa da aplicação do Termo de Consentimento Livre e Esclarecido, pois o trabalho tratou-se de uma pesquisa retrospectiva com uso de prontuários hospitalares da própria instituição (HETDLGF - Hospital de Emergência e Trauma Dom Luiz Gonzaga Fernandes), portanto, nenhum paciente ou profissional foi submetido a qualquer entrevista.

Foram analisados 524 prontuários de pacientes atendidos no Hospital de Emergência e Trauma Dom Luiz Gonzaga Fernandes (HETDLGF), na cidade de Campina Grande-PB (Brasil), no período de março de 2017 a fevereiro de 2018 (período sem o serviço de residência) e março de 2018 a fevereiro de 2019 ( $1^{\circ}$ ano do serviço de residência).

Foram incluídos no estudo os prontuários dos pacientes sem restrição de sexo, etnia e faixa etária, internados e submetidos a procedimento cirúrgico como fratura de face, infecções e/ou alterações maxilofaciais e lesão em tecido mole da face, e que apresentaram o prontuário devidamente preenchido, com as informações relevantes para análise do estudo nos anos descritos. Foram excluídos da amostra os prontuários que continham dados incompletos, que estavam rasurados ou ainda àquelas cujas informações não estavam compreensíveis.

A coleta de dados foi realizada junto aos setores de arquivo das enfermarias, livros de procedimentos cirúrgicos e o Serviço de Arquivo Médico e Estatística (SAME) do devido nosocômio. Foi utilizada uma ficha impressa em papel sulfite A4 (Anexo A) e caneta para coleta dos dados com as seguintes variáveis: data de nascimento, idade, gênero, data de admissão, data da cirurgia, data de alta, período de internação hospitalar, fratura em face, infecções e/ou alterações maxilofaciais, lesão em tecido mole da face tratado em centro cirúrgico e o tratamento realizado. A coleta de dados foi realizada por um único examinador.

Os resultados foram compilados em um banco de dados organizado em planilhas do Microsoft Excel® (Microsoft Corporation, USA) e posteriormente analisados no software SPSS ${ }^{\circledR}$ for Windows 25.0 (Statistical Package for Social Sciences $^{\mathrm{TM}}$; Inc., Chicago, USA). A estatística descritiva foi utilizada para caracterização da amostra.

As variáveis quantitativas foram descritas por medidas de tendência central e variabilidade. As variáveis qualitativas foram representadas pela distribuição de frequências. Entre as variáveis quantitativas, o tempo de internação e o tempo de espera, a partir da observação da curtose e assimetria, apresentaram distribuição normal. A partir disso, o teste T comparando as médias foi utilizado para verificar a diferença estatística entre os grupos (antes e depois da implementação da residência) e a média do tempo de internação e o tempo de espera para a realização da cirurgia com $\mathrm{p}<0.05$.

\section{Resultados}

Dos 524 prontuários, oito foram excluídos. Aplicando-se os critérios de inclusão pré-estabelecidos, obteve-se uma amostra final de 516 prontuários médicos dos pacientes atendidos pelo Serviço de Cirurgia e Traumatologia do Hospital de 
Emergência e Trauma Dom Luiz Gonzaga Fernandes de Campina Grande-PB. Constatou-se que 416 pacientes (80.6\%) eram do sexo masculino e 100 (19.4\%) eram do sexo feminino. Além disso, 236 pacientes atendidos (45.7\%) foram avaliados no período que antecedeu a implementação do Serviço de Residência, obtendo uma média de 19,6 cirurgias mensais. 280 pacientes (54.3\%) foram atendidos após a implementação do Serviço, com uma média de 23,3 cirurgias por mês. A divisão desses atendimentos por mês avaliado está disposta na Figura 1 e a apresentação da equipe e serviço na Figura 2.

Figura 1. Comparação do número de pacientes atendidos por mês, antes e após a implementação da Residência.

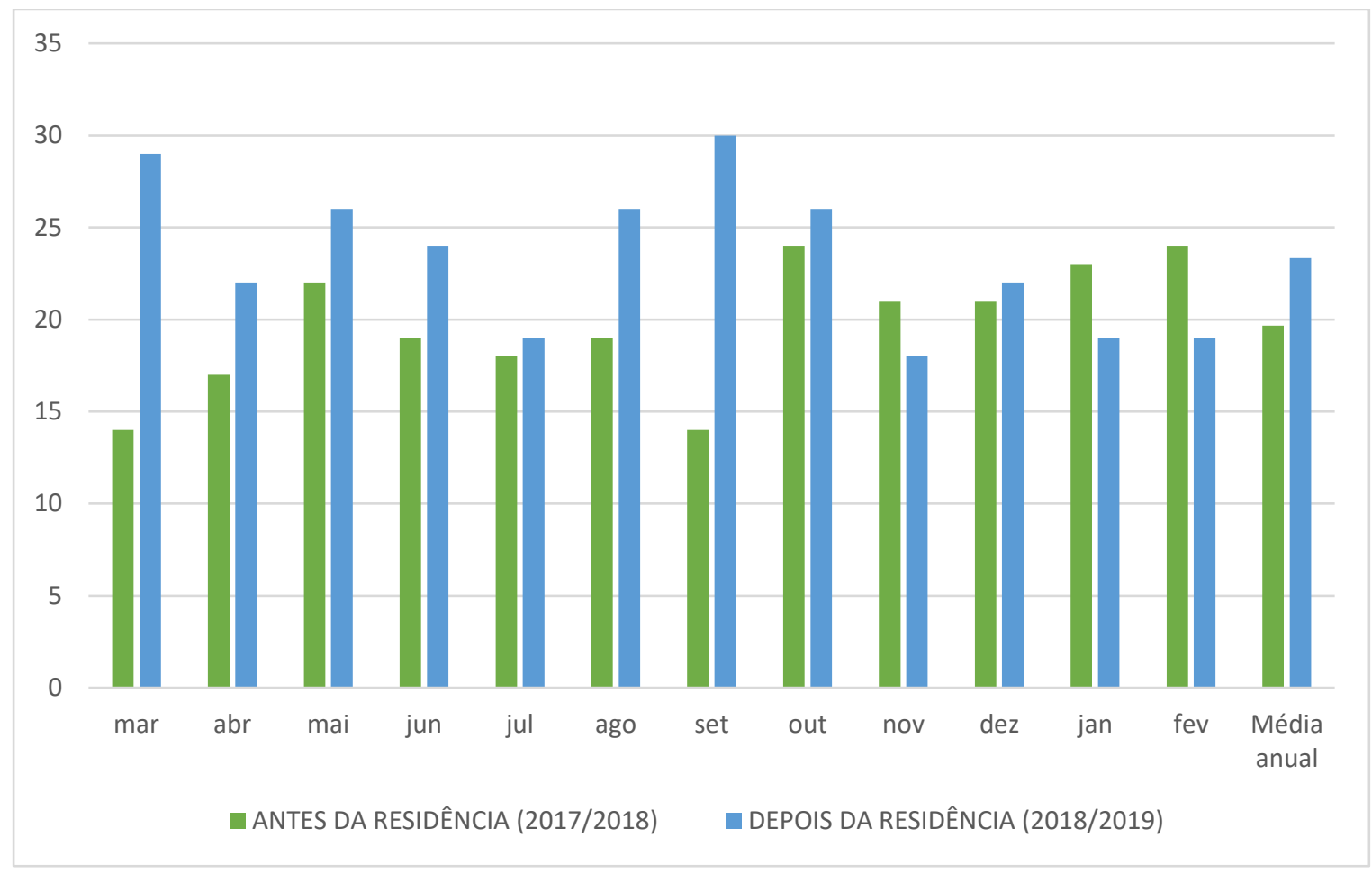

Fonte: Autoria própria (2021).

Dentre os prontuários avaliados neste estudo, pôde-se averiguar sobre o sexo, a média e o desvio-padrão da idade, tempo de espera para a cirurgia e o tempo total de internação, além do tipo de fratura, tipo de infecção e presença ou não de lesão em tecido mole da face que estão dispostas na Tabela 1, divididas entre o período avaliado dos pacientes antes da Residência e após a implementação. 
Tabela 1. Dados sobre os pacientes com a distinção entre os dois grupos avaliados.

\begin{tabular}{|c|c|c|c|}
\hline & $\begin{array}{c}\text { Total } \\
(n=516)\end{array}$ & $\begin{array}{l}\text { Antes da } \\
\text { residência } \\
(n=236)\end{array}$ & $\begin{array}{l}\text { Depois da } \\
\text { residência } \\
(n=280)\end{array}$ \\
\hline \multicolumn{4}{|l|}{ Sexo } \\
\hline Masculino & $416(80.6 \%)$ & $203(86 \%)$ & $213(76.1 \%)$ \\
\hline Feminino & $100(19.4 \%)$ & $33(14 \%)$ & $67(23.9 \%)$ \\
\hline Idade, em anos ( \pm d.p.) & $31.3( \pm 15.4)$ & $33.0( \pm 15.2)$ & $29,8( \pm 15.4)$ \\
\hline Tempo de espera para a cirurgia, em dias ( \pm d.p.) & $5.2( \pm 4.2)$ & $6.6( \pm 4.1)$ & $4.1( \pm 3.9)$ \\
\hline Tempo total de internação, em dias ( \pm d.p.) & $6.8( \pm 4.2)$ & $8.2( \pm 3.9)$ & $5.6( \pm 4.0)$ \\
\hline \multicolumn{4}{|l|}{ Tipo de Fratura, de acordo com a região anatômica } \\
\hline Zigomático & $191(34.2 \%)$ & $111(38.4 \%)$ & $80(29.6 \%)$ \\
\hline Mandíbula & $131(23.4 \%)$ & $62(21.4 \%)$ & $69(25.6 \%)$ \\
\hline Maxila & $119(21.3 \%)$ & $64(22.1 \%)$ & $55(20.4 \%)$ \\
\hline OPN & $80(14.3 \%)$ & $43(14.9 \%)$ & $37(13.7 \%)$ \\
\hline Frontal & $15(2.7 \%)$ & $2(0.7 \%)$ & $13(4.8 \%)$ \\
\hline NOE & $12(2.1 \%)$ & $2(0.7 \%)$ & $10(3.7 \%)$ \\
\hline Dento alveolar & $11(2.0 \%)$ & $5(1.7 \%)$ & $6(2.2 \%)$ \\
\hline \multicolumn{4}{|l|}{ Infecções e/ou alterações maxilofaciais } \\
\hline Celulite & $24(42.1 \%)$ & $10(52.6 \%)$ & $14(36,9 \%)$ \\
\hline Abscesso & $17(29.9 \%)$ & $5(26.3 \%)$ & $12(31.5 \%)$ \\
\hline Exposição de placa & $13(22.8 \%)$ & $4(21.1 \%)$ & $9(23.7 \%)$ \\
\hline Tumor & $3(5.2 \%)$ & - & $3(7.9 \%)$ \\
\hline \multicolumn{4}{|l|}{ Lesão em tecido mole tratado em centro cirúrgico } \\
\hline Presente & $59(100 \%)$ & $14(23.8 \%)$ & $45(76.2 \%)$ \\
\hline
\end{tabular}

Legenda: OPN: Ossos próprios do nariz; NOE: Naso-órbito-etmoidal.

Fonte: Autoria própria (2021).

Quanto aos procedimentos realizados antes e após a implementação do Serviço em CTBMF, foram destacados alguns procedimentos mais prevalentes, dos quais são indispensáveis na rotina do Residente na área e do próprio Cirurgião BucoMaxilo-Facial. Dentre os 624 procedimentos, 287 (46\%) foram vistos antes da residência e 337 (54\%) após a implementação desta. O procedimento mais observado foi a fixação com placas e parafusos, contabilizando um total de 289 procedimentos $(46,7 \%)$. Na Tabela 2, estão dispostos os procedimentos realizados, suas respectivas porcentagens distinguindo-os entre os dois grupos avaliados. 
Tabela 2. Tipos de procedimentos realizados antes e após a implementação do Serviço de Residência.

\begin{tabular}{lccc}
\hline & $\begin{array}{c}\text { Total } \\
(\mathbf{n = 5 1 6})\end{array}$ & $\begin{array}{c}\text { Antes da } \\
\text { residência } \\
(\mathbf{n = 2 3 6 )}\end{array}$ & $\begin{array}{c}\text { Depois da } \\
\text { residência } \\
(\mathbf{n = 2 8 0})\end{array}$ \\
\hline Procedimentos realizados & & & \\
\hline Fixação com placas e parafusos & $289(46.3 \%)$ & $150(52.3 \%)$ & $139(41.2 \%)$ \\
\hline Redução fechada da fratura & $100(16 \%)$ & $54(18.9 \%)$ & $46(13.6 \%)$ \\
\hline Sutura & $59(9.5 \%)$ & $14(4.9 \%)$ & $45(13.3 \%)$ \\
\hline TNA & $73(11.7 \%)$ & $38(13.3 \%)$ & $35(10.4 \%)$ \\
\hline Drenagem & $42(6.7 \%)$ & $15(5.2 \%)$ & $27(8.1 \%)$ \\
\hline Contenção & $19(3.0 \%)$ & $8(2.8 \%)$ & $11(3.2 \%)$ \\
\hline Remoção de placa & $12(2.0 \%)$ & $4(1.4 \%)$ & $8(2.3 \%)$ \\
\hline Cirurgia ortognática & $8(1.3 \%)$ & - & $8(2.3 \%)$ \\
\hline Enxerto ósseo & $5(0.8 \%)$ & $1(0.3 \%)$ & $4(1.2 \%)$ \\
\hline Patologias & $5(0.8 \%)$ & $1(0.3 \%)$ & $4(1.2 \%)$ \\
\hline Remoção de corpo estranho & $4(0.6 \%)$ & $1(0.3 \%)$ & $3(1.0 \%)$ \\
\hline TNP & $4(0.6 \%)$ & $1(0.3 \%)$ & $3(1.0 \%)$ \\
\hline Artrocentese & $2(0.3 \%)$ & - & $2(0.6 \%)$ \\
\hline Redução de luxação de ATM & $1(0.2 \%)$ & - & $1(0.3 \%)$ \\
\hline Remoção de mí́ase & $1(0.2 \%)$ & - & $1(0.3 \%)$ \\
\hline TOTAL DE PROCEDIMENTOS & & & \\
\hline
\end{tabular}

Legenda: TNA: tamponamento nasal anterior; TNP: tamponamento nasal posterior; ATM: articulação temporomandibular. Fonte: Autoria própria.

Com relação ao tempo de espera para a cirurgia e ao tempo de internação hospitalar, foram encontrados valores estatisticamente significativos, tendo uma diminuição média de 2,5 dias do tempo de espera e uma redução de 2,6 dias no tempo médio total de internação hospitalar. A Tabela 3 evidencia os dados sobre o tempo de espera para a cirurgia e o tempo de internação, distinguindo-os entre os grupos avaliados.

Tabela 3. Relação entre o tempo de espera para a cirurgia e de internação entre os grupos avaliados.

\begin{tabular}{cccccc}
\hline & Grupo & N & Média Diária & $\begin{array}{c}\text { Desvio } \\
\text { Padrão }\end{array}$ & p \\
\hline \multirow{2}{*}{ Tempo de espera } & Antes da residência & 236 & 6.6 & 4.1 \\
\cline { 2 - 5 } & Depois da residência & 280 & 4.1 & 3.9 & \multirow{2}{*}{0.000} \\
\hline \multirow{2}{*}{ Tempo de internação } & Antes da residência & 236 & 8.2 & 4.0 \\
\cline { 2 - 5 } & Depois da residência & 280 & 5.6 & \multirow{2}{*}{4.0} \\
\hline
\end{tabular}

Fonte: Autoria própria.

Utilizando o estudo de Porto, Cavalcanti; Forte, (2021) como base, onde o valor médio de pacientes vítimas de acidente de trânsito por internação é de R \$ 500,41 e a média de permanência em dias é de 1,18, conseguimos simular um valor em que o HETDLGF pode ter economizado. Dividindo o valor médio de pacientes vítimas de acidente de trânsito por internação 
( $\mathrm{R} \$ 500,41)$ pela média de permanência em dias $(1,18)$, obtém-se um valor de $\mathrm{R} \$ 424,07$. Ou seja, a cada dia de permanência de um paciente vítima de trânsito, têm-se um gasto de R \$ 424,07. Logo, sabendo que nosso estudo conseguiu constatar uma redução do tempo de internação hospitalar de 8,2 para 5,6 dias, temos que ao multiplicar o gasto diário de R $\$ 424,07$ pelos 8,2 dias (antes da Residência) e 5,6 dias (depois da Residência), obtém-se um resultado de R \$ 3.477,37 e R \$ 2.374,79, respectivamente. Sendo assim, cabe inferir que baseado na literatura existente, o HETDLGF pode ter economizado um valor de R\$1.102,58 por internação após a implementação do Serviço de CTBMF, tendo em vista que o valor médio era de R \$ 500,41. A apresentação da equipe e do serviço pode ser conferido na Figura 2.

Figura 2. Parte da equipe de Cirurgia e Traumatologia Buco-Maxilo-Facial do Hospital Estadual de Emergência e Trauma Dom Luiz Gonzaga Fernandes. Campina Grande-PB, Brasil.
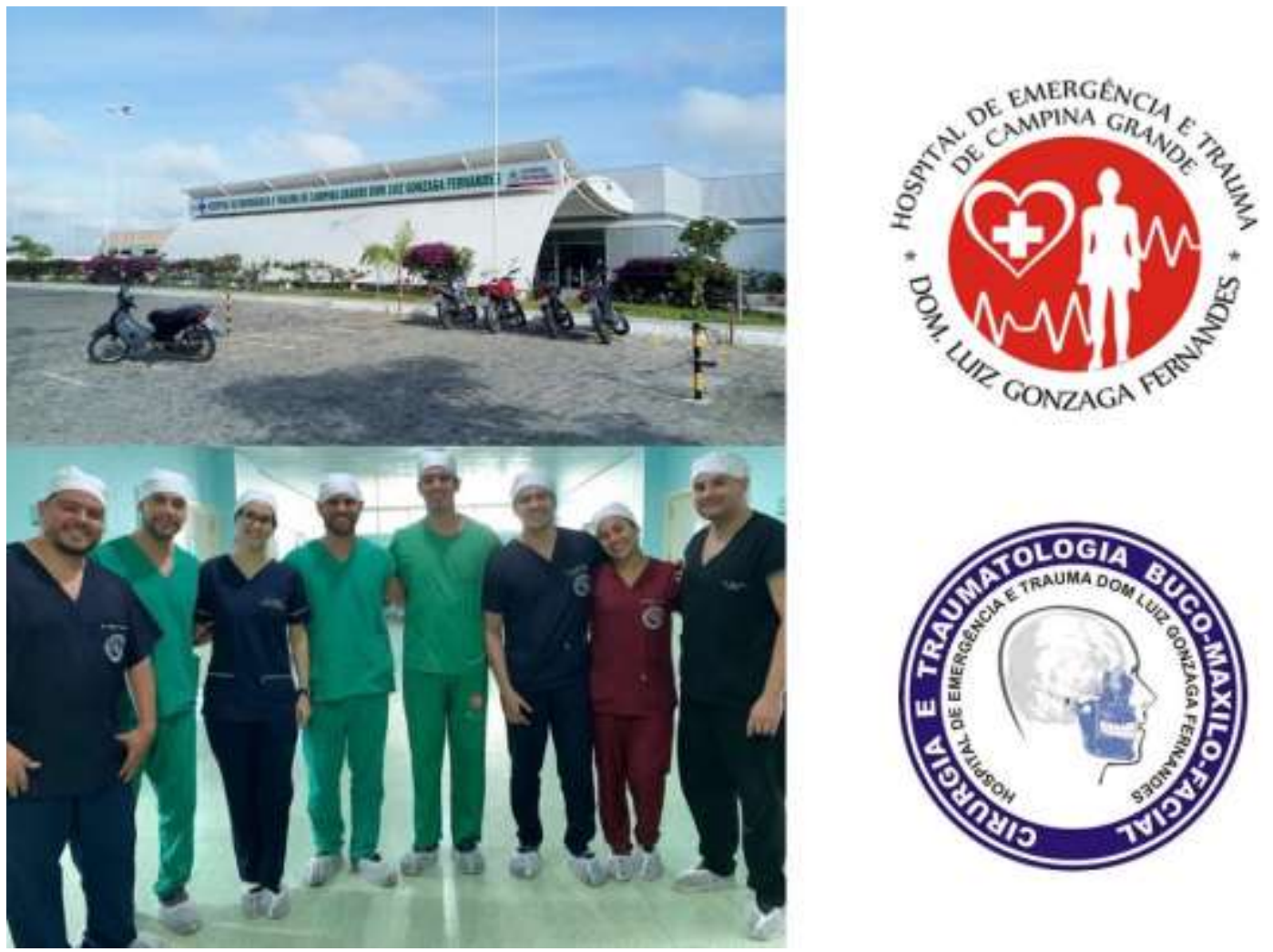

Fonte: Autoria própria (2021).

\section{Discussão}

O trauma em si pode ser considerado como o conjunto de perturbações causado subitamente por um agente físico de etiologia, natureza e extensão variadas, podendo estar situado principalmente na região de face, onde podem representar $50 \%$ de todas as mortes traumáticas (Moura, Daltro; Almeida, 2016). Nesse sentido, a divulgação de dados relacionados à epidemiologia dos traumatismos faciais e dos serviços de residência, é de extrema importância para a sociedade, a fim de proporcionar informações extremamente necessárias para a redução da incidência dos casos e desenvolvimento de medidas preventivas (Bittencourt, 2011).

O Hospital de Emergência e Trauma Dom Luiz Gonzaga Fernandes (HETDLGF) localizado na cidade de Campina Grande-PB surge como uma das principais referências em trauma para o interior da Paraíba e estados circunvizinhos (Costa, 2015). Sendo assim, a presença de um cirurgião bucomaxilofacial faz-se necessária, uma vez que este possui competência para 
avaliar e tratar os traumas de face, as lacerações na região maxilofacial, o sangramento e o consequente comprometimento das vias aéreas (Riboli, Pinheiro Siqueira, De Conto, 2016).

Portanto, sabendo que o cirurgião bucomaxilofacial é o único especialista que passa por um programa de Residência necessário para instituir atendimento craniomaxilofacial completo, abrangendo o conhecimento médico-odontológico e a capacidade necessária para lidar com a reconstrução de todos os elementos da face, o atual estudo objetivou avaliar uma amostra de prontuários dos pacientes operados pelo Serviço de Cirurgia Buco-Maxilo-Facial (CBMF) do HTEDLGF, na cidade de Campina Grande - PB (Brasil), no período de março de 2017 a fevereiro de 2018 (período sem o Serviço de Residência) e março de 2018 a fevereiro de 2019 ( $1^{\circ}$ ano do Serviço de Residência), com o intuito de avaliar o impacto da implementação deste Serviço no hospital e gerar dados concretos para futuras pesquisas.

Na presente pesquisa pôde ser observado que a amostra total do estudo referente aos anos mencionados, foi de 516 prontuários de pacientes, sendo que 416 pacientes (80,6\%) eram do sexo masculino e 100 (19,4\%) do sexo feminino. A proporção homem/ mulher encontrada foi de 4,1:1, coincidindo com o trabalho feito no extremo sul catarinense de Baroni, Celina, Cunha, (2019), que constatou a grande desproporção de atendimento entre os sexos. No atual estudo, as lesões faciais acometeram, predominantemente, o sexo masculino (80,6\%), sendo quase que impossível diferir dos estudos da literatura (Chalya et al., 2011; Samieirad, et al., 2017).

Em relação à faixa etária, a média de idade dos pacientes atendidos no presente estudo entre o período de funcionamento da residência foi de 29,8 anos, corroborando com estudos recentes em que a incidência dos pacientes atendidos ocorreu no intervalo de 21 aos 30 anos de idade (Mayrink, Avila, Belonia, 2018; Leozir et al., 2019). Estudos como o de Brasileiro, Vieira, Silveira (2010), mostra que embora os anos passem, a faixa etária ainda permanece estagnada entre 21 a 30 anos de idade, haja vista a maior atividade dessa faixa etária, expondo-se mais a fatores de risco e com um perfil menos prudente quanto aos hábitos de dirigir, muitas vezes inconsequente, pela própria idade.

Assim sendo, vale salientar que o trauma é um dos principais problemas públicos de saúde em todos os países, independentemente do desenvolvimento socioeconômico (Fonseca et al., 2019). Pasqual et al., (2020), em uma análise retrospectiva de 1.230 casos de trauma maxilofacial, avaliou que a mandíbula e o complexo zigomático foram os ossos mais acometidos. Em contrapartida, Baroni, Celin, Cunha, (2019), identificou em sua amostra que o osso nasal foi o mais atingido. No presente estudo, o osso mais acometido foi o zigomático, seguido da mandíbula. Essa frequência pode ser justificada porque a maioria das fraturas de mandíbula e de zigoma são resultados de agressão física e acidentes de trânsito, etiologias extremamente recorrentes na vivência do cirurgião (Zamboni et al., 2017).

Analisando a prevalência no que diz respeito à conduta cirúrgica e aos procedimentos em si, um dado nesse estudo foi bastante semelhante aos existentes na literatura, no qual o tratamento mais realizado foi a fixação das fraturas faciais com placas e parafusos em 289 (46.7\%), seguido do procedimento de redução incruenta da fratura com 100 casos (16.1\%). Esse dado corrobora com a pesquisa de Lins et al., (2018), quando se observou que o procedimento mais comum foi a redução mais fixação da fratura, em 150 casos $(66,66 \%)$. O alto índice desse procedimento cirúrgico pode ser justificado em decorrência da predominância das regiões mais afetadas no estudo, as quais necessitam de uma restauração adequada do alinhamento anatômico dos fragmentos (Costa, 2015).

Além disso, cabe inferir que 236 pacientes (45.7\%) foram avaliados no período que antecedeu a implementação do Serviço de Residência em CTBMF do HETDLGF, obtendo uma média de 19,6 cirurgias mensais e 280 pacientes (54.3\%) foram atendidos após a implementação do referido Serviço, com uma média de 23,3 cirurgias por mês. Observa-se um acréscimo de 44 cirurgias, no mais, equivalendo a mais de dois meses de cirurgias a mais em relação ao ano antecessor à implementação do Programa de Residência. Portanto, a inserção de residentes $(\mathrm{n}=2)$ no $1^{\circ}$ ano do Serviço pôde oferecer um número maior de procedimentos para os pacientes que necessitassem do atendimento, além de diminuir a sobre carga do hospital e possíveis 
gastos, tendo em vista que a internação hospitalar no Brasil em 2004 conduziu um investimento de aproximadamente $\mathrm{R} \$ 200.000,00$ para casos de vítimas de traumatismo facial (Siqueira et al., 2016).

Vale salientar que além do aumento do número das cirurgias de urgência e trauma, foi implementado novos tipos de tratamento, tais como: cirurgias ortognáticas, enucleação de cistos e tumores maxilofaciais e tratamentos das desordens da articulação temporo-mandibular (DTM), tornando-se um serviço de referência, resultando em uma oferta de novas possibilidades de tratamento e considerando uma intervenção multidisciplinar a uma demanda reprimida de pacientes. Faz-se necessário considerar, portanto, uma intervenção multidisciplinar e com novos tratamentos como uma oportunidade que visa mediar o problema com diversas áreas de saberes correlatas pretendendo êxito nas ações de saúde (Alves, Carvalho, 2014).

Além de melhorar a qualidade do atendimento hospitalar, a residência implementou atendimentos ambulatoriais e o sistema de retorno, que evita a internação precoce dos pacientes mais estáveis, diminui o tempo de internação hospitalar, bem como os gastos para a devida instituição. Ou seja, do ponto de vista socioeconômico, evitar a internação precoce e/ou prevenção do trauma também é vantajoso, pois os custos com tratamentos para pacientes vítimas de traumas giram em torno de bilhões de dólares (Siqueira et al., 2016). Deste modo, o Serviço permitiu um efetivo assistencialismo para a população de Campina-GrandePB e região, da mesma forma que pôde gerar uma economia interna para a instituição.

Um dado bastante relevante na atual pesquisa, foi o tempo médio de espera pelo paciente internado para o tratamento cirúrgico, que diminuiu de 6,6 dias para 4,1 dias após a implementação do Programa de Residência. Já o tempo médio total de internação hospitalar reduziu mais ainda após a chegada dos residentes, tendo um declínio de 8,2 dias para 5,6 dias. No estudo de Siqueira et al., (2016), foi constado que o grande impacto no SUS (Sistema Único de Saúde) dos casos de internação hospitalar se dá pela quantidade de pacientes internados no mesmo período (dias de permanência). Portanto, reduzir o tempo de internamento é tão importante tal como não gerar internamentos precoces, pois gera uma economia à instituição.

Um outro estudo de alta relevância é o de Porto, Cavalcanti, Forte, (2017), que buscou determinar o padrão de trauma maxilofacial por acidentes de trânsito e quedas em um hospital de referência. Os autores também descreveram os custos hospitalares de pacientes com fraturas faciais, buscando trazer um custo médio geral e um outro custo para os pacientes vítimas de acidentes de trânsito. Em relação aos gastos, os resultados apuraram que os custos hospitalares de pacientes com fraturas em face totalizaram $\mathrm{R} \$ 191.830,65$, com um custo médio geral de $\mathrm{R} \$ 330,96$ por paciente com trauma de face. Para os pacientes vítimas de acidentes de trânsito, o custo hospitalar total foi de $R \$ 150.635,56$, com uma média de $R \$ 500,41$ por paciente. Sobre a média de permanência de internação hospitalar entre todos os acidentados, observou-se uma permanência de 1,18 dias (gasto médio geral por internação de $\mathrm{R} \$ 330,96$ ) (Porto, Cavalcanti, Forte, 2021).

Ressalta-se ainda que o procedimento cirúrgico precoce no trauma de face oferece os melhores resultados e contribui para promover o retorno do paciente mais rapidamente às atividades profissionais e ao convívio da família (Leozir, et al., 2019). Sendo assim, cabe ao residente em formação na área de Cirurgia e Traumatologia Buco-Maxilo-Facial, compreender as demandas do Serviço e oferecer o tratamento mais rápido, eficaz e multidisciplinar, pois na própria ótica dos residentes, o ser humano tem suas complexidades e apenas um profissional não detém o conhecimento suficiente para suprir as necessidades desse indivíduo (Pascoaloti et al., 2019).

Em suma, o estudo apresenta vantagens e limitações. Baseado na literatura pertinente, ainda não foi feito nenhum estudo semelhante ao atual, sendo necessário mais estudos nesse sentido com serviços de residência em ascensão, a fim de caracterizar as reais vantagens e melhorias da implementação de um serviço público, gratuito e de qualidade. Sendo assim, cabe aos chefes de departamento avaliarem as situações de suas respectivas residências para determinar e compreender qual o papel está sendo realizado na sociedade. 


\section{Conclusão}

Pode-se concluir que a implementação de um serviço de residência em um hospital público promove um impacto positivo na melhoria da qualidade do atendimento aos pacientes internados. Além de promover um aumento do número de cirurgias, reduz o tempo de espera do paciente pelo tratamento cirúrgico, o tempo de internação hospitalar e consequentemente, os custos financeiros ao hospital.

Assim, sugere-se que pesquisas futuras sejam desenvolvidas em diversas instituições hospitalares, abrangendo não somente as residências da Cirurgia e Traumatologia Buco-Maxilo-Facial, mas sim todos os setores existentes. O reconhecimento sobre o potencial de um Serviço deve acontecer constantemente nas instituições, a fim de estimular a manutenção e potencializar as relações e cuidados em saúde.

\section{Referências}

Alves, J, \& Carvalho, P. (2014). Secure your network and endpoints with Importância do Programa de Residência Multiprofissional em Saúde da Família da Universidade Federal de Pernambuco na formação dos profissionais da área de saúde. EFDeportes. Com, Revista Digital, $18(1), 1-7$.

Baroni;, E. J., Celin;, R. A. T., \& Cunha, S. P. da. (2019). Etiologia e incidência de fraturas faciais em um hospital localizado em um município do extremo sul catarinense. Journal of the Brazilian College of Oral and Maxillofacial Surgery, 5(1), 22-29.

Bittencourt, L. B. de. (2011). Análise epidemiológica de fraturas bucomaxilo faciais em pacientes atendidos no Hospital Governador Celso Ramos Florianópolis/ Santa Catarina. In Trabalho de Conclusão de Curso apresentado na Universidade Federal de Santa Catarina. Universidade Federal de Santa Catarina.

Brasileiro, B. F., Vieira, J. M., \& Silveira, C. E. S. da. (2010). Avaliação de traumatismos faciais por acidentes motociclísticos em Aracaju/SE. Rev. Cir. Traumatol. Buco-Maxilo-Fac, 10(2), 97-104.

Chalya, P. L., Mchembe, M., Mabula, J. B., Kanumba, E. S., \& Gilyoma, J. M. (2011). Etiological spectrum, injury characteristics and treatment outcome of maxillofacial injuries in a Tanzanian teaching hospital. Journal of Trauma Management and Outcomes, $5(1), 7$.

Costa, M. C. F. da. (2015). Prevalência de traumas faciais no Hospital Regional de Emergência e Trauma Dom Luiz Gonzaga Fernandes no período de janeiro de 2013 à dezembro de 2014. In Monografia apresentada ao Curso de pós-graduação Lato Sensu. Universidade Estadual da Paraíba, Campina Grande-PB.

Falcão;, M. F. L., Segundo;, A. V. L., \& Silveira;, M. M. F. da. (2005). Epidemiológico de 1758 Fraturas Faciais Tratadas no Hospital da Restauração, Recife/PE. Rev. Cir. Traumatol. Buco-Maxilo-Fac, 5(3), 65-72.

Fonseca, E. V. da, Martins, D. F., Cardoso, R., Filho, M. R. P. S., \& Lima, L. H. F. (2019). Prevalence of mandibular fractures of a tertiary hospital, reference in trauma of São Paulo. Journal of the Brazilian College of Oral and Maxillofacial Surgery, 5(3), 34-39.

Gil, A. C (2008). Métodos e técnicas de pesquisa social. Atlas, 6.

Lee, K. (2012). Global Trends in Maxillofacial Fractures. Craniomaxillofacial Trauma \& Reconstruction, 5(4), $213-222$.

Leite Segundo, A. V., Campos, M. V. de S., \& Vasconcelos, B. C. do E. (2005). Perfil epidemiológico de pacientes portadores de fraturas faciais. Rev. Ciênc. Méd., (Campinas), 14(4), 345-350.

Leozir, J. P. J., Aurélio, M.V.B., Moraes, R. B., Júnior, C. A. D. A., Freitas, D. L. de, \& Sacchetti, R. (2021). Epidemiological study of facial injuries in Cacoal / RO, Brazil. Journal of the Brazilian College of Oral and Maxillofacial Surgery, 5(1).

Lins, M. A., Albuquerque, G. C. De, Oliveira, A. L. De, Martins, V. B., Fayad, F. T., Oliveira, M. V. de, \& Motta Júnior, J. (2018). Epidemiologia do trauma facial em um hospital do município de Manaus-Amazonas. Journal of the Brazilian College of Oral and Maxillofacial Surgery, 4(1), $28-32$.

Mayrink, G., Avila, N. G. A., \& Belonia, J. B. (2018). Levantamento epidemiológico dos traumas faciais em um hospital público em Vitória/ES (Brasil). Journal of the Brazilian College of Oral and Maxillofacial Surgery, 4(3), 42-47.

Motta, M. M. (2009). Análise epidemiológica das fraturas faciais em um hospital secundário. Rev. Bras. Cir. Plást, 24(2), 162-169.

Moura, M. T. F. L. de, Daltro, R. M., \& Almeida, T. F. de. (2016). Traumas faciais: uma revisão sistemática da literatura. RFO, Passo Fundo, 21 (3), 331-337.

Rodrigues, F. H. C., Miranda, E. S. de, Souza, V. E. de M. e, Castro, V. M. de, Oliveira, D. R. F. de, \& Leão, C. E. G. (2006). Avaliação Do Trauma Bucomaxilofacial No Hospital Maria Amélia Lins Da Fundação Hospitalar Do Estado De Minas Gerais. Rev. Soc. Bras. Cir. Plást., 21 (4), 211-216.

Paqual;, P. G., Arguello;, R. B., Dos-Santos;, K. W., Oliveira;, M. G. de, \& Caiton Heitz. (2020). Epidemiologia do trauma maxilofacial: uma análise retrospectiva de 1.230 casos. Journal of the Brazilian College of Oral and Maxillofacial Surgery, 6(1), 47-53.

Paraíba., G. da. (2019). Hospital de Trauma de Campina Grande realiza mais de 9 mil atendimentos em maio. Campina Grande. 
Research, Society and Development, v. 10, n. 8, e43510817682, 2021 (CC BY 4.0) | ISSN 2525-3409 | DOI: http://dx.doi.org/10.33448/rsd-v10i8.17682

Pascoaloti, M. I. M., Moreira, G. E., Rosa, C. F., Fernandes, L. A., \& Lima, D. C. (2019). Odontologia Hospitalar: desafios, importância, integração e humanização do tratamento. Revista Ciência Em Extensão, 15(1), 20-35.

Porto, P., Cavalcanti, Y. W.; \& Forte, F. D. (2021). Maxillofacial trauma due to traffic accidents and falls: an exploratory study of associated factors. Medicina oral, patologia oral y cirugia bucal, 26(3), e349-e356.

Riboli, R., Pinheiro Siqueira, S., \& de Conto, F. (2016). Papel do cirurgião bucomaxilofacial nas unidades de terapia intensiva. Revista Da Faculdade de Odontologia - UPF, 21(2), 267-270.

Samieirad, S., Aboutorabzade, M. R., Tohidi, E., Shaban, B., Khalife, H., Hashemipour, M. A., \& Salami, H. R. (2017). Maxillofacial fracture epidemiology and treatment plans in the Northeast of Iran: A retrospective study. Medicina Oral, Patologia Oral y Cirugia Bucal, 22(5), e616-e624.

Santos, M. E. S. M., Silva, É. K. P. da, Rocha, W. B. S. S., \& Vasconcelos, J. M. de. (2016). Perfil epidemiológico das vítimas de traumas faciais causados por acidentes motociclísticos. Rev. Cir. Traumatol. Buco-Maxilo-Fac, 16(1), 29-38.

Sahni, V. (2018). Psychological Impact of Facial Trauma. Craniomaxillofacial Trauma \& Reconstruction, 11(1), 015-020.

Siqueira, S. P., Lauxen, J. R., De Conto, F., \& Barbosa Avila, V. J. (2016). Gastos financeiros do Sistema Único de Saúde em pacientes com Traumatismo Facial. Revista de Ciências Médicas e Biológicas, 15(1), 27.

Wulkan, M., Parreira, J. G., \& Botter, D. A. (2005). Epidemiologia do trauma facial. Revista Da Associacao Medica Brasileira, 51(5), $290-295$.

Zamboni, R. A., Wagner, J. C. B., Volkweis, M. R., Gerhardt, E. L., Buchmann, E. M., \& Bavaresco, C. S. (2017). Levantamento epidemiológico das fraturas de face do Serviço de Cirurgia e Traumatologia Bucomaxilofacial da Santa Casa de Misericórdia de Porto Alegre-RS. Journal of the Brazilian College of Oral and Maxillofacial Surgery, 44(5), 491-497. 